\title{
Populus balsamifera Extract and Its Active Component Salicortin Reduce Obesity and Attenuate Insulin Resistance in a Diet-Induced Obese Mouse Model
}

\author{
Despina Harbilas, ${ }^{1,2,3,4}$ Diane Vallerand, ${ }^{1,2,3,4}$ Antoine Brault, ${ }^{1,2,3,4}$ Ammar Saleem, ${ }^{4,5}$ \\ John T. Arnason, ${ }^{4,5}$ Lina Musallam, ${ }^{1,2,3,4}$ and Pierre S. Haddad ${ }^{1,2,3,4}$ \\ ${ }^{1}$ Canadian Institutes of Health Research (CIHR) Team in Aboriginal Antidiabetic Medicines, Université de Montréal, P.O. Box 6128, \\ Downtown Station, Montréal, QC, Canada H3C 3J7 \\ ${ }^{2}$ Natural Health Products and Metabolic Diseases Laboratory, Department of Pharmacology, Université de Montréal, P.O. Box 6128, \\ Downtown Station, Montréal, QC, Canada H3C 3J7 \\ ${ }^{3}$ Institute of Nutrition and Functional Foods, Université Laval, Quebec City, QC, Canada G1V 0A6 \\ ${ }^{4}$ Montreal Diabetes Research Center, Centre de Recherche du Centre Hospitalier de l'Université de Montréal, \\ Montreal, QC, Canada H1W 4A4 \\ ${ }^{5}$ Department of Biology and Center for Research in Biopharmaceuticals and Biotechnology, University of Ottawa, Ottawa, \\ ON, Canada K1N 6N5
}

Correspondence should be addressed to Lina Musallam; l.musallam@gmail.com and Pierre S. Haddad; pierre.haddad@umontreal.ca

Received 13 February 2013; Accepted 8 April 2013

Academic Editor: Ravirajsinh N. Jadeja

Copyright (C) 2013 Despina Harbilas et al. This is an open access article distributed under the Creative Commons Attribution License, which permits unrestricted use, distribution, and reproduction in any medium, provided the original work is properly cited.

Populus balsamifera L. (BP) is a medicinal plant stemming from the traditional pharmacopoeia of the Cree of Eeyou Istchee (CEINorthern Quebec). In vitro screening studies revealed that it strongly inhibited adipogenesis in 3T3-L1 adipocytes, suggesting potential antiobesity activity. Salicortin was identified, through bioassay-guided fractionation, as the active component responsible for BP's activity. The present study aimed to assess the potential of BP and salicortin at reducing obesity and features of the metabolic syndrome, in diet-induced obese C57Bl/6 mice. Mice were subjected to high fat diet (HFD) for sixteen weeks, with BP (125 or $250 \mathrm{mg} / \mathrm{kg})$ or salicortin $(12.5 \mathrm{mg} / \mathrm{kg})$ introduced in the HFD for the last eight of the sixteen weeks. BP and salicortin effectively reduced whole body and retroperitoneal fat pad weights, as well as hepatic triglyceride accumulation. Glycemia, insulinemia, leptin, and adiponectin levels were also improved. This was accompanied by a small yet significant reduction in food intake in animals treated with BP. BP and salicortin (slightly) also modulated key components in signaling pathways involved with glucose regulation and lipid oxidation in the liver, muscle, and adipose tissue. These results confirm the validity of the CEI pharmacopoeia as alternative and complementary antiobesity and antidiabetic therapies.

\section{Introduction}

Obesity results from a variety of risk factors, including unhealthy dietary habits and a sedentary lifestyle, resulting in higher energy input than output [1]. It also increases the risk for other chronic illnesses such as type 2 diabetes (T2D) and insulin resistance (IR) [1]. Insulin resistance is characterized by a decreased ability of insulin sensitive tissues to respond to insulin action. Skeletal muscle is the principal tissue involved in glucose metabolism through insulin-dependent or exercise-sensitive glucose transport (Glut4) [2] implicating the Akt [3] and AMPK [4] pathways, respectively. These pathways are also implicated in glucose metabolism in the liver [5-7] and adipose tissue [3,8-12]. The liver is considered to be the principal tissue involved in glucose storage and production [13]. Adipose tissue synthesizes and stores fatty acids and is recognized as an endocrine organ; releasing adipokines (leptin, adiponectin) that are implicated in glucose and lipid metabolism [14-20]. Obesity not only leads to 
excessive fat storage in adipose tissue, but also to ectopic fat storage in other insulin sensitive tissues such as the muscle and liver (nonalcoholic fatty liver disease; NAFLD). This, in part, contributes to the development of insulin resistance $[21,22]$.

Several metabolic and signaling pathways are involved in perpetrating the disturbances of obesity and insulin resistance in the three main insulin-sensitive tissues. PPAR $\gamma$ is involved in the differentiation of adipose tissue; inducing lipid accumulation [23]. Other pathways are involved in lipid entry (FAT/CD36, FABP4) [24-27], lipid metabolism (SREBP-1c and FAS) [28, 29], and oxidation (ACC, CPT-1, PPAR $\alpha$, UCP pathways) [30]. The ERK pathway, involved in cell proliferation and differentiation, also seems to play an important role in both the liver (leads to NAFLD) [31] and adipose tissue $[32,33]$. The IKK $\alpha \beta$ pathway is involved in the inflammatory response characteristic of obesity and indirectly mediates insulin resistance [27].

In Canada, the Cree of Eeyou Istchee (CEI) of Eastern James Bay have a prevalence of obesity and T2D that is, respectively, at least $1.5[34,35]$ and 4 times higher [36] than the general Canadian population. This may be the consequence of major lifestyle changes (decreased physical activity and gradual adoption of nontraditional diets), as well as cultural difficulty to comply with modern T2D treatments. Our team has been working with the CEI to identify plants stemming from their traditional pharmacopoeia that could offer culturally adapted complementary and alternative treatments for obesity and T2D. As part of an ethnobotanical survey, Populus balsamifera L. (Salicaceae) (balsam poplar) was identified as a plant used by the CEI to treat a variety of symptoms associated with T2D. As part of an in vitro bioassay platform used to screen for the antidiabetic potential of CEI plants, the 3T3-L1 cell line was selected to assess glitazonelike activity and stimulation of adipogenesis. Populus balsamifera L., also known as balsam poplar, unexpectedly and potently inhibited the accumulation of intracellular triglycerides [37-39], suggesting potential antiobesity activity. This plant extract contains a number of active components, namely, salicin, salicortin, salireposide, and populoside [37]. In subsequent studies conducted in the same cell line, a bioassay-guided fractionation approach identified salicortin, a salicylate glycoside, as the principal active component of P. balsamifera responsible for the observed inhibition of adipogenesis [39]. Salicortin is abundant in poplar, willow bark, and throughout the Salicaceae family [39]. Although salicylates are well known for having anti-inflammatory properties, improving insulin sensitivity [40-42], and even having antiproliferative effects [43], antiadipogenic activity had never been ascribed prior to the studies conducted by our team. We thus introduced $P$. balsamifera extract, alongside a high-fat diet (HFD), to study the plant's ability to mitigate the development of obesity using the in vivo diet-induced obese (DIO) C57BL/6 mouse model. The results clearly demonstrated that the plant extract substantially attenuated weight gain and the development of insulin resistance [44].

In the present studies, we sought to evaluate the effectiveness of $P$. balsamifera as well as its active principle salicortin at treating obesity and insulin resistance once they have been established in the same model $[45,46]$. As previously described by other researchers [47] and discussed further below, DIO mice respond in a stratified manner to the HFD; some animals being resistant to the HFD (low respondersLR) while others show the clearcut profile of metabolic disease (high responders-HR). P. balsamifera and salicortin were thus administered to the latter DIO mice in order to determine their potential effectiveness in countering obesity and insulin resistance.

\section{Materials and Methods}

2.1. Plant Extracts. Specimens of Populus balsamifera L. (Salicaceae) were collected on CEI territory (Eastern James Bay, Quebec, Canada). Dr. Alain Cuerrier, taxonomist at the Montreal Botanical Garden, confirmed that the botanical identity and voucher specimens were deposited in the MarieVictorin herbarium of the Montreal Botanical Garden in Montreal, Canada (Mis03-49). A crude 80\% ethanolic extract of $P$. balsamifera was prepared as previously described [37]. Salicortin, the active principle of $P$. balsamifera, was produced through fractionation, isolation, and purification of the crude plant extract as previously described [39]. The structure of the purified compound was identified and confirmed by ${ }^{1} \mathrm{H}$ and ${ }^{13} \mathrm{C}$ NMR and by comparison with previously reported data. 1D- and 2D-NMR spectra were generated using an Avance 400 spectrometer (Bruker Biospin Corporation) [39].

2.2. Animals and Diets. Four-week-old male nondiabetic C57BL/6 mice (Charles River Laboratories, Saint-Constant, QC, Canada) were housed in individual cages, maintained on a $12 \mathrm{~h}$ light-dark cycle in a temperature and humiditycontrolled animal room, and given free access to food and water. Following acclimatization, the mice were divided into groups of approximately 12 mice each. Chow controls received a standard diet (SD; $18 \%$ protein content, $4.5 \%$ crude fat; Charles River Animal rodent diet) for 16 weeks. Other groups were fed a high fat diet (HFD; Bio-Serv Diet \#F3282; $60 \%$ energy from fat) for eight weeks. P. balsamifera at 125 or $250 \mathrm{mg} / \mathrm{kg}$, and salicortin at $12.5 \mathrm{mg} / \mathrm{kg}$ were incorporated in the HFD and treatments continued for an additional 8 weeks (DIO controls receiving only HFD). Balsam poplar extract studies were initiated first while the active compound was being identified, isolated, or purified. Since, salicortin composes $10 \%$ of the whole plant extract, and that the most efficient dose of balsam poplar was $125 \mathrm{mg} / \mathrm{kg}$, salicortin was administered at $12.5 \mathrm{mg} / \mathrm{kg}$. Hence, experimental groups of plant extract and salicortin are compared to distinct $\mathrm{CHOW}$ nonobese and DIO controls. However, both experimental protocols were conducted in an identical manner, and DIO controls reacted in a fully comparable fashion relative to nonobese Chow congeners in both studies. Body weight, food and water intake, as well as glycemia were measured 3 times/week during the entire study. Glycemia was measured by pricking the tail vein and by using a commercial glucometer (Accu-Check Roche, Montreal, QC, Canada). Measurements were always performed at the same time/day, in the same order and by the same person. All experimental 
protocols were approved by the animal experimentation ethics committee of the Université de Montréal and were carried out in full respect of the guidelines from the Canadian Council for the Care and Protection of Animals.

2.3. Data and Animal Segregation. The area under the curve (AUC) was calculated for parameters measured in a continuous manner throughout the study. The total AUC was then separated into two parts: fraction $1(\mathrm{~F} 1)$, representing AUC between week 0 and 4 (first month of treatment), and fraction 2 (F2) corresponding to the AUC between week 4 and 8 of plant extract administration (second month of treatment). This segregation served to determine the plant extract's temporal course of action, that is, whether early in onset (first 4 weeks), later (last 4 weeks), or present throughout the study. Once the experimental feeding protocol had been carried out, we became aware of the studies of Peyot and collaborators [47] discriminating low responders (LR) and high responders (HR) in the DIO mouse model. As discussed by these authors and observed in our own studies, pooling animals with different characteristics, such as low weight gain, weak IR and near-normal glycemia, with animals with high weight gain, frank IR, and hyperglycemia, can yield misleading results [47]. Therefore, we segregated the DIO animals based on these published criteria and analyzed our data accordingly. As expected, low responder animals exhibited a near normal metabolic profile and treatment with the plant extract or its active component essentially had little if any effect. This is positive in the sense that $P$. balsamifera extract and salicortin may have a desirable safety margin by being active only in metabolically compromised animals. This also confirms the validity of the segregation. Hence, data are presented for the effects of plant extract and active principle in HR animals only. This segregation did however reduce our sample size, hence contributing to data variability.

2.4. Surgical Procedure. At the end of the experimental protocol, mice were anesthetized with an intraperitoneal injection of $50 \mathrm{mg} / \mathrm{kg}$ pentobarbital and sacrificed by exsanguination. Livers were flushed with physiological saline, dissected, immediately placed in liquid nitrogen, and stored until further use at $-80^{\circ} \mathrm{C}$. Soleus skeletal muscle, white adipose tissue (WAT; epididymal and retroperitoneal fat pads), subscapular brown adipose tissue (BAT), and kidneys were also collected, placed in liquid nitrogen, and stored at $-80^{\circ} \mathrm{C}$ until further use.

2.5. Blood Parameters. Plasma insulin, adiponectin, and leptin were assessed by radioimmunoassay (RIA: Linco Research, St.Charles, MO, USA). To avoid interrupting dietary plant treatment and disturbing the HFD feeding pattern (hence affecting the DIO model), mice were not fasting when blood parameters were measured. Plasma levels of AST, ALT, LDH, creatinine, alkaline phosphatase, and circulating lipids (triglycerides, total cholesterol, LDL-cholesterol and HDL-cholesterol) were measured by standard clinical biochemistry assays at the Department of Biochemistry of Sainte-Justine's Children Hospital (Montreal, QC, Canada).
2.6. Tissue Triglyceride Measurement. Part of the frozen liver and muscle sections (around $100 \mathrm{mg}$ of each sample) were ground into powder under liquid nitrogen and extracted using Folch's chloroform/methanol (2:1) method [48]. Triglyceride content was quantified using a commercial kit (Randox Laboratories Ltd., UK).

2.7. Western Blot Analysis. Western blot analysis was performed on frozen liver, muscle, and WAT using the following antibodies: p-Akt (Ser 473), Akt, p-AMPK (Thr 172), AMPK, Glut4, p-ACC, ACC, FAS, FABP4, phospho p44/42 MAPK, $\mathrm{p} 44 / 42$ MAPK, $\mathrm{p}-\mathrm{IKK} \alpha \beta$, and $\beta$-actin (each at $1: 1000$ in blocking buffer incubated overnight at $4^{\circ} \mathrm{C}$; Cell Signaling Tech Inc., Danvers, MA, USA). PPAR $\alpha, \operatorname{PPAR} \gamma, \mathrm{CPT}-1$, CD36, UCP-2, and SREBP1-c were measured using a 1:200 dilution in blocking buffer and incubated either at $1 \mathrm{~h}$ room temperature (RT) or overnight (Santa Cruz Biotechnology Inc., Santa Cruz, CA, USA). The following HRP-conjugated secondary antibodies were used: anti-rabbit (1:10000; Jackson Immunoresearch Laboratories Inc., West Grove, PA, USA), anti-mouse (1: 4000; Cell Signaling Tech Inc., Danvers, MA, USA), or anti-goat (1:5000; Santa Cruz Biotechnology inc., Santa Cruz, CA, USA). Immunoreactive proteins were detected by enhanced chemiluminescence method (GE Healthcare, Baie d'Urfé, QC, Canada). Densitometric analysis was performed using NIH Image J software (version 1.42q, NIH, USA).

2.8. Statistical Analysis. Data were analyzed by one-way analysis of variance (ANOVA) with Bonferroni post hoc analysis, or by unpaired Student's $t$ test (Sigma Stat software, Jandel Scientific, San Rafael, CA, USA), as appropriate. Areas under the curve (AUC) were calculated with PRISM software (GraphPad, San Diego, CA, USA). Data are expressed as the mean \pm SEM of the indicated number of determinations. Statistical significance was set at $P<0.05$.

\section{Results}

3.1. Metabolic Profile of Responders to the High Fat Diet (DIO Controls). As anticipated in relation to recent data by Peyot et al. [47], roughly half of the mice consuming the HFD became obese and insulin resistant. Compared to Chow controls, such DIO control animals gained body weight, increased liver, BAT and WAT weights (Table $1, P<0.05$ ), and displayed hyperlipidemia (total cholesterol, LDL, HDL). These mice also exhibited increased plasma glucose and insulin levels, an enhanced leptin/adiponectin ratio (Table 2, $P<0.05$ ) as well as elevated hepatic and muscle triglyceride (TG) levels (Table 3; $P<0.05$ compared to Chow), thus confirming the presence of an insulin resistant state. Only mice displaying an altered metabolic profile at 8 weeks of HFD feeding were selected for the present study and randomized to receive P. balsamifera or its active component, salicortin, for an additional 8 weeks.

3.2. P. balsamifera and Salicortin Decrease Body Weight, Liver Weight, and Steatosis in DIO Mice. Treatment with 
TABLE 1: Effects of obesity, P. balsamifera, and salicortin treatments on body and organ weights at sacrifice.

\begin{tabular}{|c|c|c|c|c|c|}
\hline & DIO & $\begin{array}{l}\text { P. balsamifera } \\
125 \mathrm{mg} / \mathrm{kg}\end{array}$ & $\begin{array}{l}\text { P. balsamifera } \\
250 \mathrm{mg} / \mathrm{kg}\end{array}$ & DIO & $\begin{array}{c}\text { Salicortin } \\
12.5 \mathrm{mg} / \mathrm{kg}\end{array}$ \\
\hline Body Weight & $138 \pm 1^{\dagger}$ & $120 \pm 7^{*}$ & $131 \pm 3$ & $142 \pm 2^{\dagger}$ & $130 \pm 3^{\S}$ \\
\hline Retroperitoneal Fat Pad & $229 \pm 12^{\dagger}$ & $190 \pm 28$ & $209 \pm 20$ & $242 \pm 13^{\dagger}$ & $218 \pm 10$ \\
\hline Epididymal Fat Pad & $77 \pm 3^{\dagger}$ & $98 \pm 17$ & $103 \pm 5^{*}$ & $97 \pm 2$ & $149 \pm 11^{\S}$ \\
\hline Brown Adipose tissue & $189 \pm 14^{\dagger}$ & $\begin{array}{c}136 \pm 22 \\
P=0.057\end{array}$ & $168 \pm 14$ & $200 \pm 6^{\dagger}$ & $170 \pm 11^{\S}$ \\
\hline Liver Weight & $167 \pm 6^{\dagger}$ & $108 \pm 12^{*}$ & $122 \pm 10^{*}$ & $166 \pm 9^{\dagger}$ & $118 \pm 7^{\S}$ \\
\hline Total Kidney & $111 \pm 4^{\dagger}$ & $93 \pm 3^{*}$ & $104 \pm 4$ & $104 \pm 2$ & $101 \pm 3$ \\
\hline
\end{tabular}

Measurements were obtained after 16 weeks of treatment with either standard diet (Chow), HFD (DIO), and for the last 8 of the 16 weeks with HFD in combination with $P$. balsamifera at 125 or $250 \mathrm{mg} / \mathrm{kg}$, or with the active salicortin at $12.5 \mathrm{mg} / \mathrm{kg}$. All values are expressed as a percentage of respective Chow controls (reference set at $100 \%$ ) and represent the mean \pm SEM. The number of animals for each group for the P. balsamifera protocol was: CHOW ( $n=12)$; DIO $(n=8)$; P. balsamifera $125(n=5)$; P. balsamifera $250(n=7)$; and for the salicortin protocol: CHOW $(n=12)$; DIO $(n=7)$; salicortin $(n=9) .{ }^{\dagger}$ denotes DIO significantly different as compared to Chow (unpaired Student's $t$ test; $P<0.05$ ). ${ }^{*}$ denotes significantly different as compared to respective DIO (one way ANOVA, Bonferroni post hoc test; $P<0.05$ ). ${ }^{\S}$ denotes significantly different as compared to respective DIO (unpaired Student's $t$ test; $P<0.05$ ).

TABLE 2: Effects of obesity, P. balsamifera, and salicortin treatments on systemic parameters at sacrifice.

\begin{tabular}{|c|c|c|c|c|c|}
\hline & DIO & $\begin{array}{l}\text { P. balsamifera } \\
125 \mathrm{mg} / \mathrm{kg}\end{array}$ & $\begin{array}{l}\text { P. balsamifera } \\
250 \mathrm{mg} / \mathrm{kg}\end{array}$ & $\mathrm{DIO}$ & $\begin{array}{c}\text { Salicortin } \\
12.5 \mathrm{mg} / \mathrm{kg}\end{array}$ \\
\hline Glucose $(\mathrm{mmol} / \mathrm{L})$ & $135 \pm 14^{\dagger}$ & $105 \pm 8$ & $121 \pm 10$ & $121 \pm 6^{\dagger}$ & $114 \pm 6$ \\
\hline Insulin $(\mathrm{ng} / \mathrm{mL})$ & $3056 \pm 1074^{\dagger}$ & $450 \pm 238^{*}$ & $\begin{array}{c}832 \pm 423 \\
(P=0.052)\end{array}$ & $1035 \pm 150^{\dagger}$ & $272 \pm 62^{\S}$ \\
\hline Leptin (ng/mL) & $211 \pm 28^{\dagger}$ & $108 \pm 18^{*}$ & $\begin{array}{c}145 \pm 9 \\
(P=0.051)\end{array}$ & $246 \pm 19^{\dagger}$ & $197 \pm 13^{\S}$ \\
\hline Adiponectin $(\mu \mathrm{g} / \mathrm{mL})$ & $70 \pm 3^{\dagger}$ & $78 \pm 10$ & $\begin{array}{c}82 \pm 5 \\
(P=0.054)\end{array}$ & $97 \pm 5$ & $101 \pm 6$ \\
\hline Leptin/adiponectin ratio & $304 \pm 37^{\dagger}$ & $138 \pm 16^{*}$ & $181 \pm 16^{*}$ & $248 \pm 12^{\dagger}$ & $196 \pm 18^{\S}$ \\
\hline TG (mmol/L) & $99 \pm 8$ & $80 \pm 10$ & $82 \pm 8$ & $118 \pm 10$ & $106 \pm 14$ \\
\hline $\mathrm{LDL}(\mathrm{mmol} / \mathrm{L})$ & $391 \pm 31^{\dagger}$ & $355 \pm 37$ & $307 \pm 42$ & $344 \pm 20^{\dagger}$ & $207 \pm 22^{\S}$ \\
\hline $\mathrm{HDL}(\mathrm{mmol} / \mathrm{L})$ & $141 \pm 8^{\dagger}$ & $112 \pm 17$ & $125 \pm 8$ & $157 \pm 10^{\dagger}$ & $137 \pm 7$ \\
\hline Total cholesterol (mmol/L) & $180 \pm 9^{\dagger}$ & $152 \pm 14$ & $151 \pm 12$ & $203 \pm 11^{\dagger}$ & $151 \pm 7^{\S}$ \\
\hline $\operatorname{ALT}(\mathrm{U} / \mathrm{L})$ & $281 \pm 39^{\dagger}$ & $229 \pm 47$ & $271 \pm 77$ & $341 \pm 123^{\dagger}$ & $157 \pm 27$ \\
\hline AST (U/L) & $172 \pm 30^{\dagger}$ & $153 \pm 22$ & $137 \pm 22$ & $163 \pm 27^{\dagger}$ & $86 \pm 5^{\S}$ \\
\hline Creatinine (U/L) & $184 \pm 50$ & $491 \pm 160$ & $557 \pm 138$ & $276 \pm 113$ & $136 \pm 30$ \\
\hline Alkaline phosphatase (U/L) & $115 \pm 20$ & $85 \pm 15$ & $100 \pm 9$ & $106 \pm 9$ & $88 \pm 17$ \\
\hline $\mathrm{LDH}(\mathrm{U} / \mathrm{L})$ & $341 \pm 83^{\dagger}$ & $137 \pm 16^{\S}$ & $193 \pm 61$ & $376 \pm 156^{\dagger}$ & $154 \pm 38$ \\
\hline
\end{tabular}

Measurements were obtained after 16 weeks of treatment with either standard diet (Chow), HFD (DIO), and for the last 8 of the 16 weeks with HFD in combination with P. balsamifera at 125 or $250 \mathrm{mg} / \mathrm{kg}$, or with the active salicortin at $12.5 \mathrm{mg} / \mathrm{kg}$. All values are expressed as a percentage of their respective Chow controls (reference set at 100\%) and represent the mean \pm SEM. The number of animals for each group for the P. balsamifera protocol was: CHOW $(n=12)$; DIO $(n=8)$; P. balsamifera $125(n=5)$; P. balsamifera $250(n=7)$; and for the salicortin protocol: CHOW $(n=12)$; DIO $(n=7)$; salicortin $(n=9)$. ${ }^{\dagger}$ denotes DIO significantly different as compared to Chow (unpaired Student's $t$ test; $P<0.05$ ). ${ }^{*}$ denotes significantly different as compared to respective DIO (one way ANOVA, Bonferroni post hoc test; $P<0.05$ ). ${ }^{\S}$ denotes significantly different as compared to respective DIO (unpaired Student's $t$ test; $P<0.05$ ).

TABLE 3: Effects of obesity, P. balsamifera, and salicortin treatments on hepatic and muscular triglyceride accumulation.

\begin{tabular}{lccccc}
\hline & DIO & P. balsamifera $125 \mathrm{mg} / \mathrm{kg}$ & P. balsamifera $250 \mathrm{mg} / \mathrm{kg}$ & DIO & Salicortin $12.5 \mathrm{mg} / \mathrm{kg}$ \\
\hline Liver TG Levels $(\mathrm{mg} / \mathrm{g}$ total liver) & $930 \pm 65^{\dagger}$ & $436 \pm 146^{*}$ & $521 \pm 116^{*}$ & $1084 \pm 180^{\dagger}$ & $559 \pm 93^{\S}$ \\
Muscle TG levels $(\mu \mathrm{g} / \mathrm{mg})$ & $223 \pm 54^{\dagger}$ & $342 \pm 81$ & $267 \pm 38$ & $230 \pm 32^{\dagger}$ & $219 \pm 24$ \\
\hline
\end{tabular}

The colorimetric dosage of TG levels in both the liver and muscle was determined using a commercial kit (Randox Laboratories ltd). Measurements were obtained after 16 weeks of treatment with either standard diet (Chow), HFD (DIO), and for the last 8 of the 16 weeks with HFD in combination with $P$. balsamifera at 125 or $250 \mathrm{mg} / \mathrm{kg}$, or with the active salicortin at $12.5 \mathrm{mg} / \mathrm{kg}$. All values are expressed as percentage of respective Chow (reference set at $100 \%$ ) and represent the mean \pm SEM. The number of animals for each group for the P. balsamifera protocol was: CHOW $(n=12)$; DIO $(n=8) ;$. balsamifera 125 $(n=5)$; P. balsamifera $250(n=7)$; and for the salicortin protocol: CHOW $(n=12)$; DIO $(n=7)$; salicortin $(n=9) .{ }^{\dagger}$ denotes DIO significantly different as compared to Chow (unpaired Student's $t$ test; $P<0.05$ ). ${ }^{*}$ denotes significantly different as compared to respective DIO (one way ANOVA, Bonferroni post hoc test; $P<0.05)$. ${ }^{\S}$ denotes significantly different as compared to respective DIO (unpaired Student's $t$ test; $P<0.05$ ). 


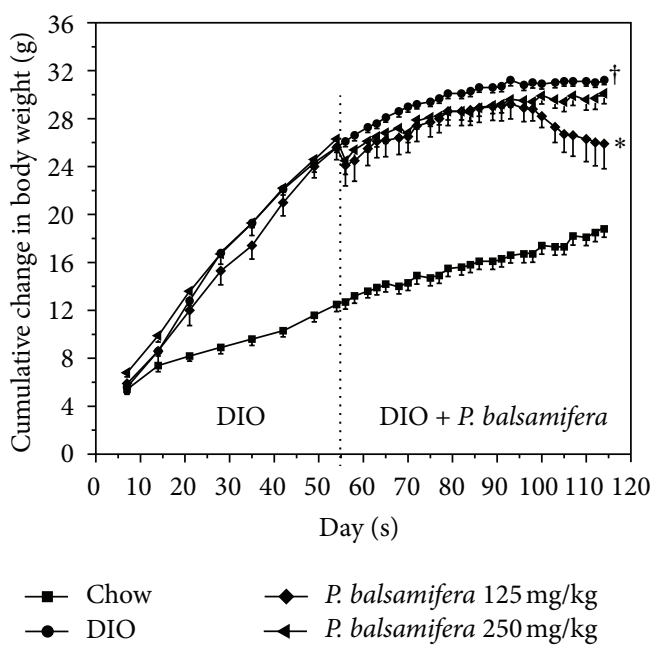

(a)

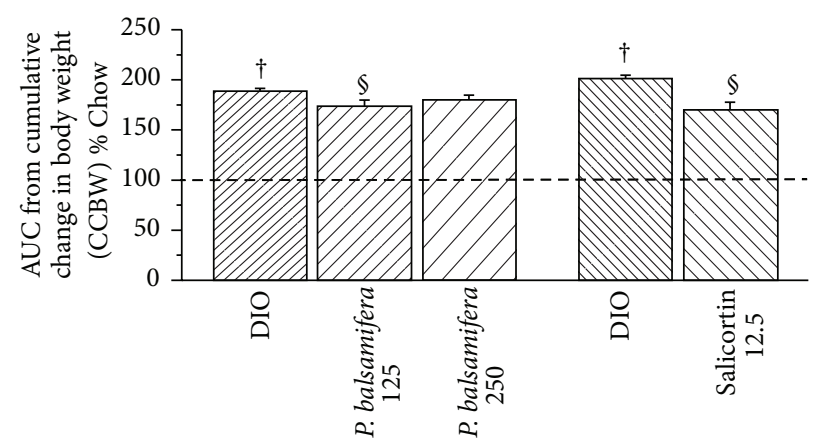

(c)

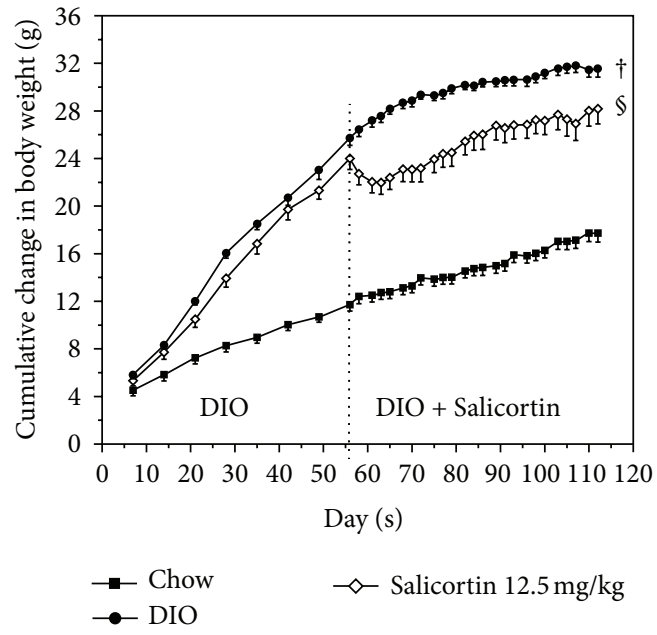

(b)

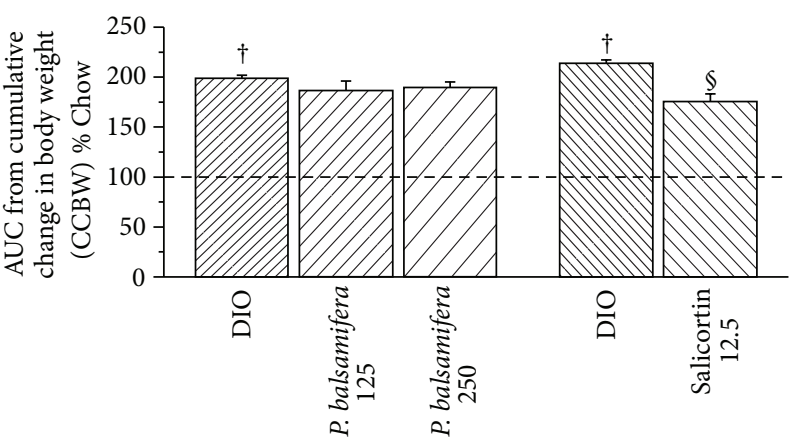

(d)

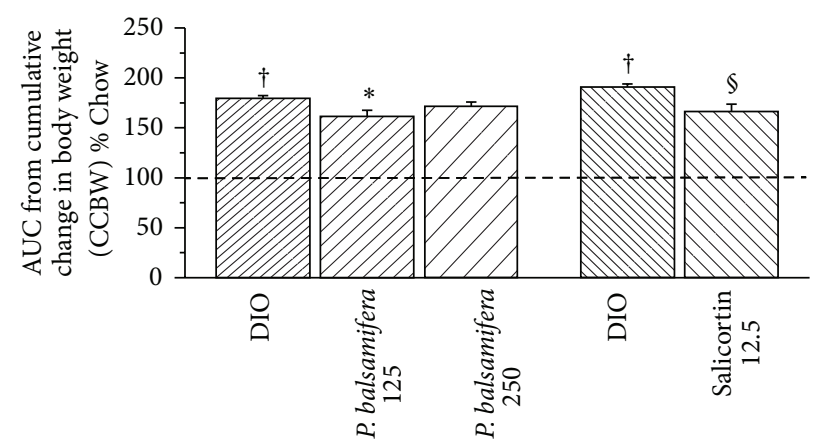

(e)

FIGURE 1: Cumulative changes in body weight (CCBW) in C57BL/6 mice treated with either standard diet (Chow), HFD (DIO), and (a) HFD in combination with P. balsamifera at 125 or $250 \mathrm{mg} / \mathrm{kg}$, or (b) HFD in combination with salicortin. Area under the curve (AUC) of CCBW for (c) total 8-week treatment period, (d) first 4 weeks of treatment (F1), and (e) second 4 weeks of treatment (F2). As mentioned, C57BL/6 mice were administered either standard diet (Chow), HFD (DIO), and for the last 8 of the 16 weeks a HFD in combination with $P$. balsamifera at 125 or $250 \mathrm{mg} / \mathrm{kg}$, or with Salicortin $12.5 \mathrm{mg} / \mathrm{kg}$. All values are mean \pm SEM. Fraction 1 (F1) consists in the AUC between week 0 and 4 , and fraction 2 (F2) corresponds to the AUC between week 4 and 8 of administration of the plant extract. The number of animals for the crude plant extract protocol was CHOW $(n=12)$, DIO $(n=8)$, P. balsamifera $125(n=5)$, and P. balsamifera $250(n=7)$; and for the salicortin protocol: CHOW $(n=12)$, DIO $(n=7)$, salicortin $(n=9) .{ }^{\dagger}$ denotes DIO significantly different as compared to Chow (unpaired Student's $t$ test; $P<0.05)$. * denotes significantly different as compared to respective DIO (one way ANOVA; $P<0.05$ ). ${ }^{\S}$ denotes significantly different as compared to respective DIO (unpaired Student's $t$ test; $P<0.05$ ).

P. balsamifera (at $125 \mathrm{mg} / \mathrm{kg}$ ) significantly decreased body weight. This decrease reached $13 \%$ at sacrifice when compared to DIO controls $(P<0.05$; Table 1$)$. When taking into account continuous measurements of cumulative changes in body weight (CCBW; Figures 1(a) and 1(b)), the area under the curve (AUC) was lowered by $8 \%(P<0.05$; Figure $1(\mathrm{c}))$ with $125 \mathrm{mg} / \mathrm{kg}$ of $P$. balsamifera. This effect was gradual, beginning within the first month $(F 1=6 \%$ reduction; N.S.; Figure $1(\mathrm{~d}))$, but becoming more pronounced in the second month of the treatment $(F 2=10 \%$ decrease; $P<0.05$; Figure $1(\mathrm{e}))$. 


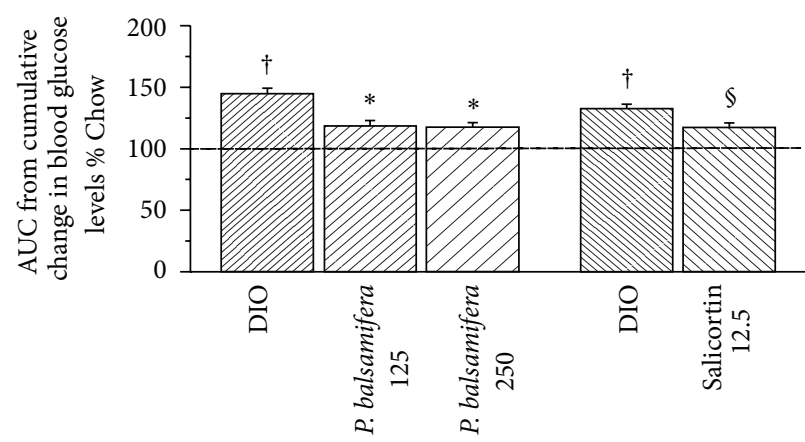

(a)

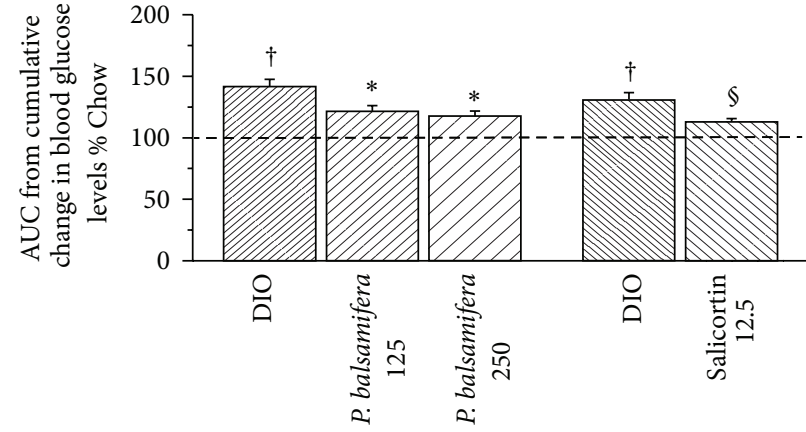

(b)

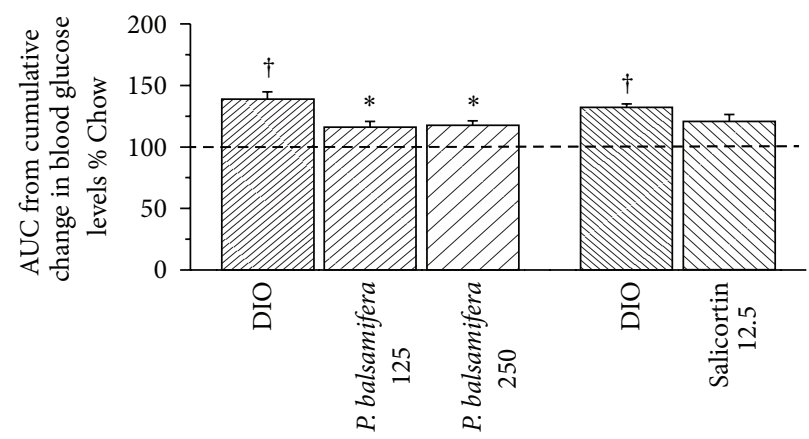

(c)

Figure 2: Area under the curve (AUC) of cumulative changes in blood glucose levels for (a) total 8-week treatment period, (b) first 4 weeks of treatment (F1), and (c) second 4 weeks of treatment (F2). C57BL/6 mice were administered either standard diet (Chow), HFD (DIO), and for the last 8 of the 16 weeks a HFD in combination with $P$. balsamifera at 125 or $250 \mathrm{mg} / \mathrm{kg}$, or with salicortin $12.5 \mathrm{mg} / \mathrm{kg}$. All values are mean \pm SEM. Fraction 1 (F1) consists in the AUC between week 0 and 4, and fraction 2 (F2) corresponds to the AUC between week 4 and 8 of administration of the plant extract. The number of animals for the crude plant extract protocol was CHOW $(n=12)$, DIO $(n=8), P$. balsamifera $125(n=5)$, and P. balsamifera $250(n=7)$; and for the salicortin protocol: CHOW $(n=12)$, DIO $(n=7)$, salicortin $(n=9)$. ${ }^{\dagger}$ denotes DIO significantly different as compared to Chow (unpaired Student's $t$ test; $P<0.05$ ). ${ }^{*}$ denotes significantly different as compared to respective DIO (one way ANOVA; $P<0.05$ ). ${ }^{\S}$ denotes significantly different as compared to respective DIO (unpaired Student's $t$ test; $P<0.05)$.

Animals receiving $250 \mathrm{mg} / \mathrm{kg}$ of $P$. balsamifera exhibited a similar pattern of effects, albeit without reaching statistical significance. Mice fed the active compound, salicortin, also displayed a significant decrease in body weight amounting to an $8 \%$ drop at sacrifice $(P<0.05$; Table 1$)$ and a $15 \%$ decrease in total AUC of CCBW $(P<0.05$; Figure 1(c)). Interestingly, its effect was immediate with $18 \%$ decrease in the F1 AUC $(P<0.05$; Figure $1(\mathrm{~d}))$ that continued into the second month, albeit slightly less prominently $(F 2=13 \%$; $P<0.05$; Figure 1(e)).

Concomitantly, epididymal fat pad weight increased, whereas retroperitoneal fat pad weight was smaller when animals were treated with either dose of balsam poplar $(9 \%-$ $17 \%$ reduction) or with salicortin (10\% decrease) than in those receiving $\mathrm{HFD}$ alone, although the latter changes failed to reach statistical significance (Table $1 ;$ N.S). In contrast, the drop in liver weight was significant in the three aforementioned treated groups (decrease by $27 \%-35 \%$; $P<0.05$ versus DIO controls; Table 1). Consistent with these results, hepatic triglyceride (TG) content was also reduced by $44 \%$ to $53 \%$ in the treated animals in comparison to DIO controls $(P<0.05$; Table 3). Muscle triglycerides, however, were not significantly altered by any of the treatments (N.S.; Table 3).
It must be noted that a weak anorexic effect was observed in animals receiving $P$. balsamifera at $125 \mathrm{mg} / \mathrm{Kg}$ dose. Indeed, the AUC of cumulative food intake of this group was significantly reduced by $6 \%$ as compared to DIO congeners $(P<$ 0.05 , data not shown). No such effect was observed with the higher dose of P. balsamifera or with salicortin.

3.3. P. balsamifera and Salicortin Improve Insulin Sensitivity, While only the Active Principle Modulates Lipidemia in DIO Mice. Along with body weight changes, P. balsamifera (at 125 and $250 \mathrm{mg} / \mathrm{kg}$ ) and salicortin improved insulin sensitivity, albeit with slightly different profiles. Firstly, continuous measurement of glycemia showed that both doses of whole plant extract as well as the active principle significantly reduced total AUC by $17 \%-18 \%$ and by $11 \%$, respectively. Although $P$. balsamifera and salicortin effects were rather rapid in onset, the effect of the whole plant was constant throughout the treatment period $(F 1=F 2)$, whereas that of the active principle decreased with time $(F 1=14 \%$, $P<0.05$ versus DIO controls; F2 = 9\%, N.S.; Figures 2(b) and 2(c)). At sacrifice, glycemia of the three treatment groups was reduced as compared to their respective controls, albeit not in a statistically significant manner (Table 2). 
Secondly, insulinemia diminished by $85 \%$ with balsam poplar at $125 \mathrm{mg} / \mathrm{Kg}(P<0.05)$ and by $73 \%$ with $250 \mathrm{mg} / \mathrm{Kg}$ $(P=0.052)$ as well as with salicortin $(P<0.05)$ in comparison to DIO controls (Table 2). Thirdly, the two doses of $P$. balsamifera decreased leptin/adiponectin ratio by $41 \%-$ $54 \%$ as compared to congeners receiving HFD alone $(P<$ 0.05 ; Table 2). Salicortin also significantly decreased this ratio, although to a lesser extent (by 21\%; $P<0.05$; Table 2). In terms of the circulating lipid profile, only the salicortin treated group exhibited significantly lowered total plasma cholesterol and LDL levels, which were reduced by $25 \%$ and $40 \%$, respectively (Table $2 ; P<0.05$ ) as compared to the DIO controls. Altogether, these findings illustrate an improvement in insulin sensitivity when balsam poplar or its active principle are added to the HFD.

Finally, P. balsamifera and salicortin tended to normalize several systemic parameters of toxicity, although this did not reach statistical significance, except in the case of AST levels for salicortin $(P<0.05$; Table 2$)$ and LDH levels for $P$. balsamifera at $125 \mathrm{mg} / \mathrm{Kg}(P<0.05$; Table 2$)$.

\subsection{P. balsamifera Tends to Increase Skeletal Muscle Glut4 and} Improves Components Related to Muscle Lipid Oxidation without Affecting the Akt and AMPK Pathways, Whereas Salicortin Tends to Increase Akt Phosphorylation and Activates p44/42 $M A P K$. Despite the significant reduction of overall glycemia exerted by the plant extract and its active principle, analysis of protein components involved in muscle glucose homeostasis did not exhibit any statistically significant changes. There was a tendency for Glut4 expression to increase in animals treated with P. balsamifera at $125 \mathrm{mg} / \mathrm{kg}$, (Table 4; N.S. balsam poplar versus corresponding DIO controls). Similarly, insulin-dependent Akt phosphorylation tended to increase in animals fed with salicortin, although data variability precluded any definitive interpretation of these results. The insulin-independent AMPK pathway remained more clearly unchanged.

In contrast, components involved in muscle lipid homeostasis showed evidence of improvement with balsam poplar treatment. Indeed, P. balsamifera at $125 \mathrm{mg} / \mathrm{kg}$ more than doubled muscle PPAR $\alpha$ expression levels (Table 4; $137 \%$ increase compared to DIO $P<0.05)$. When looking at components involved in muscle fatty acid oxidation and synthesis, again only the plant extract seemed to act on such pathways, by tending to increase phosphorylated ACC levels and to normalize FAS levels back down to Chow levels (Table 4; N.S. compared to DIO controls). The p44/42 MAPK pathway linked to exercise and insulin stimulation was significantly activated with salicortin (Table $4, P<0.05$ ), and showed a tendency to do so with the plant extract at $250 \mathrm{mg} / \mathrm{kg}$ (Table 4, N.S.).

3.5. The Effects of P. balsamifera and Salicortin on Liver Components of Glucose and Lipid Homeostasis. Both doses of P. balsamifera significantly increased hepatic phosphorylated Akt in HFD-fed animals (Table 4; increases by $111 \%$ and $87 \%$ for 125 and $250 \mathrm{mg} / \mathrm{kg}$ groups, respectively; $P<0.05$ compared to DIO controls), while the active principle showed only a slight tendency to do so ( $22 \%$ increase). A number of parameters related to hepatic lipid homeostasis or inflammation showed interesting tendencies, but none of these effects reached statistical significance. In all cases, tendencies were more pronounced with the 125 than the $250 \mathrm{mg} / \mathrm{kg}$ dose of P. balsamifera. Notably, PPAR $\alpha$ appeared to be increased by both balsam poplar and the active principle, while CPT-1 seemed to be increased only by the plant extract (Table 4 , N.S.). As for IKK $\alpha \beta$ it appeared to be affected only by the plant extract, exhibiting a decrease of $43 \%$ and $30 \%$ with 125 and $250 \mathrm{mg} / \mathrm{kg}$ doses, respectively (Table 4; N.S.).

3.6. The Effect of P. balsamifera and Salicortin on Adipose Tissue Components of Glucose and Lipid Homeostasis. P. balsamifera at $125 \mathrm{mg} / \mathrm{kg}$ showed a strong tendency to increase phosphorylated Akt levels in adipose tissue (Table 4; increase by $65 \%, P=0.068$ compared to DIO controls). Likewise, CPT-1 expression in animals treated with the plant extract at $250 \mathrm{mg} / \mathrm{kg}$ exhibited a strong tendency to be enhanced (Table 4; increase by $47 \%, P=0.079$ compared to DIO), whereas the active principle had a similar albeit much weaker effect on this parameter (Table 4; $11 \%$ increase; N.S.). In contrast, FABP4 was clearly and significantly increased by P. balsamifera at both doses $(54 \%$ and $60 \%$ at 125 and $250 \mathrm{mg} / \mathrm{kg}$, respectively, Table $4, P<0.05$ compared to DIO controls), while salicortin showed only a slight tendency to do so (16\%, N.S., Table 4;). Salicortin and balsam poplar showed a tendency to normalize PPAR $\gamma$ and phosphorylated p44/42 MAPK to levels similar to those observed in Chow animals (Table 4). Other components failed to show any significant changes in plant or active-principle treated treated animals compared to their respective DIO controls.

\section{Discussion}

According to the World Health Organization (WHO), 75\% of the world population still relies on traditional medicine for primary health care needs and this often involves crude preparations of medicinal plants [49]. In the Canadian province of Quebec, regional health authorities assigned to the CEI are currently considering the usefulness of Cree traditional medicine, notably its associated pharmacopoeia, to deal with several health concerns such as type 2 diabetes; a condition that has reached epidemic proportions in the region [36]. Our group has been working since 2003 with communities and health authorities in CEI to build the scientific evidence base in support of this initiative. An ethnobotanical study was conducted in collaboration with CEI Elders and healers that identified several plants used to treat diabetes symptoms $[37,50,51]$. One of these was Populus balsamifera L. (Salicaceae) or balsam poplar. The plant did not demonstrate much antidiabetic potential in in vitro bioassays; for instance, it had little effect on muscle glucose uptake [37]. However, the plant caught our attention by its complete inhibition of triglyceride accumulation and adipogenesis in the 3T3-L1 adipocyte cell line [37], suggesting potential therapeutic usefulness against obesity. Salicortin, a salicylate glycoside, abundant in poplar, willow bark, as well as throughout the Salicaceae family, was identified through bioassay-guided fractionation as the constituent of 
TABLE 4: Effects of obesity, P. balsamifera, and salicortin treatments on tissue components involved in glucose and lipid homeostasis.

\begin{tabular}{|c|c|c|c|c|c|}
\hline & DIO & $\begin{array}{l}\text { P. balsamifera } \\
125 \mathrm{mg} / \mathrm{kg}\end{array}$ & $\begin{array}{l}\text { P. balsamifera } \\
250 \mathrm{mg} / \mathrm{kg}\end{array}$ & $\mathrm{DIO}$ & $\begin{array}{c}\text { Salicortin } \\
12.5 \mathrm{mg} / \mathrm{kg}\end{array}$ \\
\hline \multicolumn{6}{|l|}{ Muscle } \\
\hline Glut4 & $150 \pm 63$ & $321 \pm 174$ & $151 \pm 52$ & $70 \pm 33$ & $59 \pm 7$ \\
\hline pAkt/Akt & $214 \pm 59^{\dagger}$ & $195 \pm 47$ & $267 \pm 82$ & $96 \pm 23$ & $120 \pm 29$ \\
\hline phospho p44/42 Mapk/44/42Mapk & $178 \pm 83$ & $99 \pm 15$ & $273 \pm 84$ & $47 \pm 11$ & $146 \pm 34^{\S}$ \\
\hline $\mathrm{pAMPk} / \mathrm{AMPk}$ & $138 \pm 43$ & $79 \pm 10$ & $97 \pm 22$ & $108 \pm 16$ & $108 \pm 22$ \\
\hline $\mathrm{pACC} / \mathrm{ACC}$ & $122 \pm 28$ & $171 \pm 56$ & $157 \pm 38$ & $106 \pm 22$ & $89 \pm 26$ \\
\hline $\operatorname{PPAR} \alpha / \beta$-actine & $97 \pm 23$ & $229 \pm 50^{\S}$ & $143 \pm 44$ & $195 \pm 90$ & $162 \pm 69$ \\
\hline FAS/ $\beta$-actine & $118 \pm 30$ & $100 \pm 39$ & $77 \pm 18$ & $114 \pm 16$ & $143 \pm 29$ \\
\hline \multicolumn{6}{|l|}{ Liver } \\
\hline $\mathrm{pAkt} / \mathrm{Akt}$ & $66 \pm 14$ & $139 \pm 18^{*}$ & $124 \pm 17^{*}$ & $106 \pm 28$ & $129 \pm 37$ \\
\hline phospho p44/42 Mapk/44/42Mapk & $68 \pm 21$ & $48 \pm 21$ & $66 \pm 14$ & $109 \pm 35$ & $96 \pm 44$ \\
\hline $\mathrm{pACC} / \mathrm{ACC}$ & $105 \pm 41$ & $117 \pm 74$ & $114 \pm 62$ & $71 \pm 26$ & $77 \pm 31$ \\
\hline $\operatorname{PPAR} \alpha / \beta$-actine & $63 \pm 6^{\dagger}$ & $88 \pm 20$ & $75 \pm 13$ & $107 \pm 26$ & $147 \pm 26$ \\
\hline $\mathrm{UCP}-2 / \beta$-actine & $94 \pm 19$ & $107 \pm 29$ & $88 \pm 26$ & $151 \pm 32$ & $149 \pm 27$ \\
\hline CPT-1/ $\beta$-actine & $84 \pm 12$ & $100 \pm 8$ & $86 \pm 9$ & $86 \pm 6$ & $83 \pm 9$ \\
\hline FAS $/ \beta$-actine & $69 \pm 17$ & $69 \pm 22$ & $59 \pm 25$ & $86 \pm 27$ & $74 \pm 15$ \\
\hline SREBPl-c/ $\beta$-actine & $88 \pm 16$ & $112 \pm 27$ & $121 \pm 37$ & $109 \pm 14$ & $109 \pm 17$ \\
\hline $\mathrm{CD} 36 / \beta$-actine & $63 \pm 7^{\dagger}$ & $93 \pm 19$ & $64 \pm 3$ & $69 \pm 8^{\dagger}$ & $67 \pm 6$ \\
\hline pIKK $\alpha \beta / \beta$-actine & $108 \pm 23$ & $62 \pm 31$ & $75 \pm 12$ & $114 \pm 21$ & $129 \pm 21$ \\
\hline \multicolumn{6}{|l|}{ Adipose tissue } \\
\hline pAkt/Akt & $112 \pm 13$ & $\begin{array}{c}186 \pm 37 \\
(P=0.068)\end{array}$ & $110 \pm 15$ & $138 \pm 18$ & $109 \pm 14$ \\
\hline phospho p44/42 Mapk/44/42Mapk & $133 \pm 31$ & $103 \pm 12$ & $79 \pm 10$ & $156 \pm 23^{\dagger}$ & $128 \pm 21$ \\
\hline $\operatorname{PPAR} \gamma / \beta$-actine & $73 \pm 17$ & $74 \pm 15$ & $102 \pm 30$ & $85 \pm 12$ & $116 \pm 15$ \\
\hline $\mathrm{pACC} / \mathrm{ACC}$ & $139 \pm 46$ & $119 \pm 66$ & $157 \pm 70$ & $95 \pm 30$ & $86 \pm 20$ \\
\hline CPT-1/ $\beta$-actine & $81 \pm 9$ & $86 \pm 10$ & $\begin{array}{c}119 \pm 19 \\
(P=0.079)\end{array}$ & $89 \pm 11$ & $99 \pm 14$ \\
\hline FABP $4 / \beta$-actine & $85 \pm 11$ & $131 \pm 11^{\S}$ & $136 \pm 19^{\S}$ & $71 \pm 6$ & $83 \pm 10$ \\
\hline FAS $/ \beta$-actine & $40 \pm 5^{\dagger}$ & $33 \pm 8$ & $56 \pm 11$ & $49 \pm 7^{\dagger}$ & $45 \pm 7$ \\
\hline SREBP-1 $c / \beta$-actine & $77 \pm 6$ & $88 \pm 9$ & $96 \pm 19$ & $86 \pm 7$ & $87 \pm 11$ \\
\hline
\end{tabular}

Samples of muscle, liver, and WAT were obtained after 16 weeks of treatment with either standard diet (Chow), HFD (DIO), and for the last 8 of the 16 weeks with HFD in combination with P. balsamifera at 125 or $250 \mathrm{mg} / \mathrm{kg}$, or with the active salicortin at $12.5 \mathrm{mg} / \mathrm{kg}$. The samples were homogenized and analyzed by immunoblotting. Blots were quantified by densitometry. All values are expressed as percentage of respective Chow (reference set at 100\%) and represent the mean \pm SEM. The number of animals for each group for the P. balsamifera protocol was: CHOW $(n=12)$; DIO $(n=8)$; P. balsamifera $125(n=5)$; $P$. balsamifera $250(n=7)$; and for the salicortin protocol: CHOW $(n=12)$; DIO $(n=7)$; salicortin $(n=9)$. ${ }^{\dagger}$ denotes DIO significantly different as compared to Chow (unpaired Student's $t$ test; $P<0.05$ ). ${ }^{*}$ denotes significantly different as compared to respective DIO (one way ANOVA, Bonferroni post hoc test; $P<$ 0.05). ${ }^{\S}$ denotes significantly different as compared to respective DIO (unpaired Student's $t$ test; $P<0.05$ ).

P. balsamifera having the most potential to inhibit adipogenesis in the 3T3-L1 cell line [39]. Prior to our studies, antiadipogenic activity had never been ascribed to balsam poplar, to members of its botanical family, or to its known phytochemical constituents, such as salicortin [39]. The goal of the present study was to evaluate the effectiveness of balsam poplar and salicortin as antiobesity, antiadipogenic, and consequently antidiabetic agents in an in vivo mouse model.

The DIO mouse model was used in this study. It closely mimics human metabolic syndrome (notably obesity and insulin resistance) and requires lesser quantities of plant extracts (also, more importantly, of active principles) than larger animals for long-term studies. Indeed, in this model, a period of 8 weeks is necessary to establish obesity and insulin resistance, as confirmed in the present studies. The plant was then incorporated into the high fat diet for a further 8 weeks to fully assess its potential to treat obesity and the associated metabolic disturbances. After 16 weeks on a high fat diet, control DIO animals develop obesity, mild hyperglycemia, hyperinsulinemia, hyperleptinemia, and increased ectopic fat storage (notably hepatic steatosis), all reflecting the establishment of the metabolic syndrome and an insulin resistant state. In previous studies, a less severe 
model was used whereby animals were subjected to a HFD for only 8 weeks; P. balsamifera being administered from the onset of the HFD feeding in order to evaluate its potential to prevent obesity and its associated insulin resistant state [44]. The plant extract effectively reduced body weight gain, retroperitoneal fat pad weight, liver lipid content, as well as circulating glucose, insulin and leptin levels. It also activated pathways that were involved with glucose and lipid oxidation, as well as thermoregulation. The onset of action of the plant extract was immediate and sustained throughout its course of administration.

The results of the current study clearly demonstrate that in mice subjected to a continuous hypercaloric fatladen diet, $P$. balsamifera significantly reduced body weight, whereas its active salicortin prevented further weight gain. The plant's effect was more potent and statistically significant at $125 \mathrm{mg} / \mathrm{kg}$ than $250 \mathrm{mg} / \mathrm{kg}$. Several anthropomorphic, systemic and tissue parameters were thus examined to circumscribe the possible mechanisms of action of the plant extract and its active principle, salicortin.

A first potentially important lead came from data on cumulative food intake. Indeed, the plant extract at $125 \mathrm{mg} / \mathrm{kg}$ slightly but significantly reduced energy intake, and this was visible in the second month of treatment (F2; data not shown). This correlated well with the plant's temporal action on body weight. An initial reduction in body weight was observed upon introduction of the plant extract in the diet and may have resulted from a behavioral response to the food change. However, body weight rapidly resumed its course such that cumulative weight gain in the first month period (F1) was not significantly different among treatment groups. This contrasts with the reduced energy intake in the second month period (F2) that coincided with a significant decrease in weight gain. Such results suggested that the plant may exhibit slight appetite-modifying effects. Interestingly, these putative anorexic effects were seen only with the $125 \mathrm{mg} / \mathrm{kg}$ dose and indicate an unconventional dose-response relationship, as discussed further below. Nevertheless, such anorexic effects warrant further investigation. Notably, appetite-related hormones, such as leptin, as well as gut-brain appetite control mechanisms will need to be examined.

However, the reduction in caloric intake was weaker than the weight loss measured, roughly half to two-thirds as important when considering total or F2 AUC measurements of cumulative weight changes, respectively. In contrast, the active salicortin decreased the overall AUC of CCBW without affecting cumulative energy intake. This not only suggests different profiles of biological activity between the active principle and the plant extract, but also that other phytochemical components present within balsam poplar are contributing to its appetite-modifying effect.

On the other hand, although obesity was only partly countered by $P$. balsamifera and salicortin, systemic glucose homeostasis was more significantly improved. Indeed, continuous glycemia measurements showed that the plant and its active principle had an overall effect to reduce blood glucose variations toward normal values observed in Chowfed controls. Even more telling was the dramatic decrease of insulinemia seen with $P$. balsamifera at $125 \mathrm{mg} / \mathrm{kg}$ and with salicortin. Likewise, the leptin-to-adiponectin ratio, also reflective of insulin resistance, was essentially halved with the plant extract and decreased by 1.5 -fold with the active principle. Interestingly, salicortin also significantly improved the blood lipid profile by decreasing LDL and total cholesterol levels, whereas the plant extract had no significant impact on systemic parameters of lipid homeostasis. This again points to variations in biological activity between the crude extract and the purified active principle.

Further analysis of the major insulin responsive tissues, notably skeletal muscle, liver and adipose tissue, yielded data that highlights potential mechanisms at several levels of metabolic control. Firstly, excessive skeletal muscle TG accumulation was not corrected by P. balsamifera or salicortin treatment. In fact, if anything, balsam poplar extract at $125 \mathrm{mg} / \mathrm{kg}$ tended to increase this parameter, albeit large variations in the data preclude any definite interpretations. One possibility is that the two-month treatment was not sufficient to significantly affect muscle TG accumulation, yet improvements in muscle lipid and glucose metabolism could have been initiated. Indeed, the crude plant extract did more than double the expression of PPAR $\alpha$, which could lead to increased fatty acid oxidation [30] and improved muscle insulin sensitivity [52]. In animals receiving $125 \mathrm{mg} / \mathrm{kg}$ of the plant, muscle Glut 4 expression tended to increase and this is consistent with enhanced insulin sensitivity. In contrast, salicortin treatment only significantly affected muscle p44/42 MAPK activation, again hinting at different actions of the plant extract and its active principle.

In contrast, in the liver, P. balsamifera and salicortin treatment more than halved the elevated levels of accumulated TGs. Since hepatic steatosis is increasingly recognized as a major contributor to systemic insulin resistance $[53,54]$, this action may have played a significant role in improving systemic glucose homeostasis and insulin sensitivity. Indeed, analysis of key tissue proteins indicated that $P$. balsamifera treatment induced a doubling of liver Akt phosphorylation. Since Akt is a major component of the insulin-signaling cascade, part of the effect of balsam poplar could involve improved hepatic insulin sensitivity. Indeed, Akt inhibits glucose production and promotes glycogen deposition in the liver $[5,6,13]$. In hepatic cell lines, our group recently found that $P$. balsamifera inhibits glucose-6-phosphatase [55]. Other components also tended to be modulated by $P$. balsamifera in the liver and suggested that the plant may favor salvaging lipid metabolism. Indeed, PPAR $\alpha$ levels were increased by treatment with the plant and its active principle, this transcription factor being known to enhance fatty acid oxidation [30]. The tendency for a reduction of IKK $\alpha \beta$ by the plant treatment, on the other hand, points to a potential improvement of inflammatory components known to be involved in nonalcoholic fatty liver disease and ensuing metabolic disturbances $[27,56]$. Such effects of $P$. balsamifera and salicortin on liver lipid homeostasis and inflammation will require confirmation in future studies.

Despite large reductions in retroperitoneal fat pad weight at sacrifice, consistent with the significant reduction in body weight, such changes induced by $P$. balsamifera and salicortin 
failed to reach statistical significance due to data variability. In contrast, epididymal fat pad weight was reduced in DIO mice relative to Chow controls and this was normalized by balsam poplar extract treatment. The paradoxical decrease in epididymal fat pad weight in DIO mice may reflect the redistribution of fat towards more visceral sites in response to the high fat diet as observed by other investigators $[57,58]$.

On the other hand, obesity, especially visceral, leads to low-grade inflammation, releasing into circulation proinflammatory cytokines that contribute to the development of insulin resistance and diabetes. In addition, since both balsam poplar and salicortin belong to the salicylates family, well known for their anti-inflammatory properties, it would be of interest to evaluate the effect of these treatments on circulating proinflammatory cytokines (e.g., TNF- $\alpha$, IL-1 $\beta$, IL-6, resistin, C-reactive protein, and so on). Our group recently assessed the effects of $P$. balsamifera on TNF- $\alpha$ production in THP-1 monocytes (ATCC TIB-202). Preliminary results indicate that $P$. balsamifera displays moderate anti-inflammatory properties in LPS-stimulated THP-1 monocytes [59].

Nonetheless, analysis of adipose tissue components yielded a number of insightful results. Firstly, the tendency for $P$. balsamifera and salicortin to reduce the p44/42 ERK MAP kinase is consistent with the parallel tendency for WAT weight reductions. Indeed, the ERK pathway is involved in adipogenesis and insulin resistance $[32,60]$ and our group observed that $P$. balsamifera inhibits clonal expansion in 3T3-L1 adipocytes [38]. On the other hand, FABP-4, a lipid chaperone carrying fatty acids to cellular pathways of oxidation, was significantly increased by plant treatment. As in liver, adipose tissue Akt and CPT-1 expression also showed a strong tendency to be increased, supporting the notion that $P$. balsamifera can enhance insulin-dependent lipid oxidative pathways. The active principle showed much weaker actions on adipose tissue components, notably mild tendencies to increase FABP-4 and CPT-1 expression.

The effects of both the crude plant extract and of the active principle salicortin occurred without any overt sign of toxicity, albeit balsam poplar extract tended to increase blood creatinine whereas salicortin tended to reduce the same parameter. Future studies should assess kidney function in a more detailed manner. However, unaltered liver function parameters support the interpretation that the plant and its active salicortin are fairly innocuous. Indeed, products of this tree have been used safely for generations by several Aboriginal peoples of the Northern hemisphere [61, 62]. The inner bark of $P$. balsamifera (from which the plant extract used in the current studies was derived) is even documented as a survival food $[62,63]$.

Interestingly, the majority of metabolically and statistically significant changes were obtained with the lower dose of $125 \mathrm{mg} / \mathrm{kg}$ of $P$. balsamifera, whereas the larger $250 \mathrm{mg} / \mathrm{kg}$ dose exerted lesser or no effects. Such counterintuitive doseresponse relationships are not uncommon with polymolecular drug mixtures. Synergistic and antagonistic interactions may occur between the phytochemical components, yielding unconventional dose-response profiles [64]; for instance, observing an anorexic effect at the $125 \mathrm{mg} / \mathrm{kg}$ dose but not at that of $250 \mathrm{mg} / \mathrm{kg}$. Such interactions are also supported by the aforementioned differences in the biological activity profile between the crude plant extract and salicortin. It is conceivable that other components in the crude extract may complement salicortin's activity.

Indeed, the action of the active principle alone on continuously measured parameters (body weight and blood glucose) appeared to wane with time, since effects were more pronounced in the first month of administration (F1) than in the second (F2). This may limit the use of the active principle at this dose and may have contributed to mask effects on protein components in insulin-sensitive tissues. Further studies need to be conducted in order to determine if this apparent time-dependent decline in activity develops at any dosage, and if so, with what time course. Nevertheless, salicortin has a sufficiently promising biological profile in DIO mice to warrant further studies potentially leading to clinical assessments.

In summary, P. balsamifera and salicortin exerted significant weight-reducing properties in obese, insulin resistant mice in the face of continued HFD feeding. Part of the plant extract's effect appears to emanate from a putative weak anorexic effect that will need to be defined, taking into consideration the loss of effect with higher doses. The plant and the active principle had even more profound beneficial effects on systemic glucose homeostasis and indirect indices of insulin sensitivity. Analysis of tissue components involved in glucose and lipid homeostasis uncovered several potential lead mechanisms in key insulin responsive organs such as skeletal muscle, liver, and adipose tissue. Generally, components involved in insulin-dependent lipid oxidative pathways were most prominently and coordinately modulated in animals treated with the plant extract. Such actions would favor the "wastage" of energy derived from excess lipids consumed through the HFD, thereby reducing the negative metabolic impact of obesity. This is highly relevant for Aboriginal populations like the CEI whose rapid changes in dietary habits over the last decades also involve a higher intake of lipid-enriched calorie-dense foods. It is noteworthy that salicortin did not always activate the same pathways and to the same degree as the plant extract, suggesting that other plant constituents in the crude extract may also participate in beneficial biological activity toward metabolic disease.

In conclusion, the present studies confirm the high potential of $P$. balsamifera as a complementary treatment derived from CEI traditional medicine, which can help combat the devastating effects of obesity, often leading to type 2 diabetes. Having identified salicortin as an important active principle in vitro-its anti-obesity and mild antidiabetic effects having also been validated by the present in vivo treatment study-it can now be considered as a valuable tool to ensure the quality and efficacy of $P$. balsamifera preparations. Salicortin can also serve as a template to develop novel therapeutic agents for the treatment of obesity and type 2 diabetes. Additional studies should further clarify the mode of action of the plant and its active principle. This will pave the way toward clinical studies designed to determine if $P$. balsamifera and salicortin can be used in a safe and efficacious manner, alongside conventional medical treatments, for the treatment of metabolic diseases. 


\section{Conflict of Interests}

The authors declare no conflict of interests.

\section{Acknowledgments}

A Team Grant from the Canadian Institutes of Health Research (CTP-79855) to Pierre S. Haddad and John T. Arnason funded these studies. Jonathan Ferrier (Arnason laboratory, University of Ottawa) is gratefully acknowledged for preparing the crude extracts of $P$. balsamifera. Very special thanks are due to Smally Petawabano, Laurie Petawabano, Charlie Coon, Sophie Coon, Mabel Gunner, Abel Mark, Kathleen Mark, Elizabeth Coon Come, Harriett Matoush, Sandy Matoush, Rene Coon, and Emma Coon Come from the Cree Nation of Mistissini, and Abraham Mamianskum, Agnes Kawapit, Andrew Natachequan, Anne Sandy, Eliza George Mamianskum, Eliza Kawapit, James Kawapit, Jeanny Masty, John Petagumskum Sr., Lucy Rupert, Maggie Natachequan, and Mathew Natachequan from Whapmagoostui First nation, as well as to 41 other Cree Elders of both nations who kindly agreed to be interviewed. They made this paper possible by allowing us to use, for the purposes of this research, their knowledge relating to medicinal plants transmitted to them by their Elders. Their trust has also enabled a useful exchange between Indigenous knowledge and Western science.

\section{References}

[1] M. M. Robinson and X. Zhang, The World Medicines Situation 2011, Traditional Medicines: Global Situation, Issues and Challenges, World Health Organization, Geneva, Switzerland, 2011.

[2] H. Wallberg-Henriksson and J. R. Zierath, "GLUT4: a key player regulating glucose homeostasis? Insights from transgenic and knockout mice (review)," Molecular Membrane Biology, vol. 18, no. 3, pp. 205-211, 2001.

[3] A. R. Saltiel and C. R. Kahn, "Insulin signalling and the regulation of glucose and lipid metabolism," Nature, vol. 414, no. 6865, pp. 799-806, 2001.

[4] E. J. Kurth-Kraczek, M. F. Hirshman, L. J. Goodyear, and W. W. Winder, " 5 ' AMP-activated protein kinase activation causes GLUT4 translocation in skeletal muscle," Diabetes, vol. 48, no. 8, pp. 1667-1671, 1999.

[5] M. Bouskila, M. F. Hirshman, J. Jensen, L. J. Goodyear, and K. Sakamoto, "Insulin promotes glycogen synthesis in the absence of GSK3 phosphorylation in skeletal muscle," American Journal of Physiology, vol. 294, no. 1, pp. E28-E35, 2008.

[6] K. F. Petersen, G. W. Cline, D. P. Gerard, I. Magnusson, D. L. Rothman, and G. I. Shulman, "Contribution of net hepatic glycogen synthesis to disposal of an oral glucose load in humans," Metabolism, vol. 50, no. 5, pp. 598-601, 2001.

[7] J. T. Hwang, M. S. Lee, H. J. Kim et al., "Antiobesity effect of ginsenoside Rg3 involves the AMPK and PPAR- $\gamma$ signal pathways," Phytotherapy Research, vol. 23, no. 2, pp. 262-266, 2009.

[8] A. Kimura, S. Mora, S. Shigematsu, J. E. Pessin, and A. R. Saltiel, "The insulin receptor catalyzes the tyrosine phosphorylation of caveolin-1," The Journal of Biological Chemistry, vol. 277, no. 33, pp. 30153-30158, 2002.
[9] R. Augustin, "The protein family of glucose transport facilitators: It's not only about glucose after all," IUBMB Life, vol. 62, no. 5, pp. 315-333, 2010.

[10] B. Thorens and M. Mueckler, "Glucose transporters in the 21st century," American Journal of Physiology, vol. 298, no. 2, pp. E141-E145, 2010.

[11] L. G. D. Fryer, A. Parbu-Patel, and D. Carling, "The anti-diabetic drugs rosiglitazone and metformin stimulate AMP-activated protein kinase through distinct signaling pathways," The Journal of Biological Chemistry, vol. 277, no. 28, pp. 25226-25232, 2002.

[12] D. G. Hardie, S. A. Hawley, and J. W. Scott, "AMP-activated protein kinase-development of the energy sensor concept," Journal of Physiology, vol. 574, no. 1, pp. 7-15, 2006.

[13] R. C. Nordlie, J. D. Foster, and A. J. Lange, "Regulation of glucose production by the liver," Annual Review of Nutrition, vol. 19, pp. 379-406, 1999.

[14] N. Al-Daghri, W. A. Bartlett, A. F. Jones, and S. Kumar, "Role of leptin in glucose metabolism in type 2 diabetes," Diabetes, Obesity and Metabolism, vol. 4, no. 3, pp. 147-155, 2002.

[15] K. W. Williams, M. M. Scott, and J. K. Elmquist, "From observation to experimentation: leptin action in the mediobasal hypothalamus," American Journal of Clinical Nutrition, vol. 89, no. 3, pp. 985S-990S, 2009.

[16] J. J. Díez and P. Iglesias, "The role of the novel adipocyte-derived hormone adiponectin in human disease," European Journal of Endocrinology, vol. 148, no. 3, pp. 293-300, 2003.

[17] J. Nedvídková, K. Smitka, V. Kopský, and V. Hainer, "Adiponectin, an adipocyte-derived protein," Physiological Research, vol. 54, no. 2, pp. 133-140, 2005.

[18] F. Vasseur, F. Leprêtre, C. Lacquemant, and P. Froguel, "The genetics of adiponectin," Current Diabetes Reports, vol. 3, no. 2, pp. 151-158, 2003.

[19] N. Rasouli and P. A. Kern, "Adipocytokines and the metabolic complications of obesity," Journal of Clinical Endocrinology and Metabolism, vol. 93, no. 11, pp. s64-s73, 2008.

[20] R. V. Considine, M. K. Sinha, M. L. Heiman et al., "Serum immunoreactive-leptin concentrations in normal-weight and obese humans," The New England Journal of Medicine, vol. 334, no. 5, pp. 292-295, 1996.

[21] S. Kumar, Obesity and Diabetes, John Wiley \& Sons, 2009.

[22] G. I. Shulman, "Cellular mechanisms of insulin resistance," The Journal of Clinical Investigation, vol. 106, no. 2, pp. 171-176, 2000.

[23] S. R. Farmer, "Regulation of PPARgamma activity during adipogenesis," International Journal of Obesity, vol. 29, supplement 1, pp. S13-S16, 2005.

[24] G. Endemann, L. W. Stanton, K. S. Madden, C. M. Bryant, R. T. White, and A. A. Protter, "CD36 is a receptor for oxidized low density lipoprotein," The Journal of Biological Chemistry, vol. 268, no. 16, pp. 11811-11816, 1993.

[25] A. C. Nicholson, S. Frieda, A. Pearce, and R. L. Silverstein, "Oxidized LDL binds to CD36 on human monocyte-derived macrophages and transfected cell lines. Evidence implicating the lipid moiety of the lipoprotein as the binding site," Arteriosclerosis, Thrombosis, and Vascular Biology, vol. 15, no. 2, pp. 269-275, 1995.

[26] C. A. Baxa, R. S. Sha, M. K. Buelt et al., "Human adipocyte lipidbinding protein: purification of the protein and cloning of its complementary DNA," Biochemistry, vol. 28, no. 22, pp. 86838690, 1989.

[27] M. C. Arkan, A. L. Hevener, F. R. Greten et al., "IKK- $\beta$ links inflammation to obesity-induced insulin resistance," Nature Medicine, vol. 11, no. 2, pp. 191-198, 2005. 
[28] T. Porstmann, B. Griffiths, Y. L. Chung et al., "PKB/Akt induces transcription of enzymes involved in cholesterol and fatty acid biosynthesis via activation of SREBP," Oncogene, vol. 24, no. 43, pp. 6465-6481, 2005.

[29] J. M. Ntambi, M. Miyazaki, J. P. Stoehr et al., "Loss of stearoylCoA desaturase-1 function protects mice against adiposity," Proceedings of the National Academy of Sciences of the United States of America, vol. 99, no. 17, pp. 11482-11486, 2002.

[30] N. Chen, R. Bezzina, E. Hinch et al., "Green tea, black tea, and epigallocatechin modify body composition, improve glucose tolerance, and differentially alter metabolic gene expression in rats fed a high-fat diet," Nutrition Research, vol. 29, no. 11, pp. 784-793, 2009.

[31] N. C. Chavez-Tapia, N. Mendez-Sanchez, and M. Uribe, "Role of nonalcoholic fatty liver disease in hepatocellular carcinoma," Annals of Hepatology, vol. 8, supplement 1, pp. S34-S39, 2009.

[32] F. Bost, M. Aouadi, L. Caron, and B. Binétruy, "The role of MAPKs in adipocyte differentiation and obesity," Biochimie, vol. 87, no. 1, pp. 51-56, 2005.

[33] E. Donzelli, C. Lucchini, E. Ballarini et al., "ERK1 and ERK2 are involved in recruitment and maturation of human mesenchymal stem cells induced to adipogenic differentiation," Journal of Molecular Cell Biology, vol. 3, no. 2, pp. 123-131, 2011.

[34] WHO, Obesity Prevalence in the Aboriginal Canadian Population, 2004.

[35] WHO, Obesity Prevalence in the Canadian Population, 2004.

[36] D. Dannenbaum and E. Kuzmina, "J. T. clinical management of diabetes in Eeyou Istchee-2009," in Internal Report For Healthcare Workers, Bay rBoHaSSoJ, Ed., Public Health Report Series 3 on Diabetes, Quebec, Canada, 2010.

[37] D. Harbilas, L. C. Martineau, C. S. Harris et al., "Evaluation of the antidiabetic potential of selected medicinal plant extracts from the Canadian boreal forest used to treat symptoms of diabetes: part II," Canadian Journal of Physiology and Pharmacology, vol. 87, no. 6, pp. 479-492, 2009.

[38] L. C. Martineau, J. Hervé, A. Muhamad et al., "Anti-adipogenic activities of Alnus incana and Populus balsamifera bark extracts, part I: sites and mechanisms of action," Planta Medica, vol. 76, no. 13, pp. 1439-1446, 2010.

[39] L. C. Martineau, A. Muhammad, A. Saleem et al., "Antiadipogenic activities of alnus incana and populus balsamifera bark extracts, part II: bioassay-guided identification of actives salicortin and oregonin," Planta Medica, vol. 76, no. 14, pp. 15191524, 2010.

[40] J. K. Kim, Y. J. Kim, J. J. Fillmore et al., "Prevention of fatinduced insulin resistance by salicylate," The Journal of Clinical Investigation, vol. 108, no. 3, pp. 437-446, 2001.

[41] M. Yuan, N. Konstantopoulos, J. Lee et al., "Reversal of obesityand diet-induced insulin resistance with salicylates or targeted disruption of Ikk $\beta$," Science, vol. 293, no. 5535, pp. 1673-1677, 2001.

[42] L. Zheng, S. J. Howell, D. A. Hatala, K. Huang, and T. S. Kern, "Salicylate-based anti-inflammatory drugs inhibit the early lesion of diabetic retinopathy," Diabetes, vol. 56, pp. 337-345, 2007.

[43] B. Subramanian, A. Nakeff, K. Tenney, P. Crews, L. Gunatilaka, and F. Valeriote, "A new paradigm for the development of anticancer agents from natural products," Journal of Experimental Therapeutics and Oncology, vol. 5, no. 3, pp. 195-204, 2006.

[44] D. Harbilas, A. Brault, D. Vallerand et al., "Populus balsamifera L. (Salicaceae) mitigates the development of obesity and improves insulin sensitivity in a diet-induced obese mouse model," Journal of Ethnopharmacology, vol. 141, pp. 1012-1020, 2012.

[45] R. Buettner, J. Schölmerich, and L. C. Bollheimer, "High-fat diets: modeling the metabolic disorders of human obesity in rodents," Obesity, vol. 15, no. 4, pp. 798-808, 2007.

[46] S. Collins, T. L. Martin, R. S. Surwit, and J. Robidoux, "Genetic vulnerability to diet-induced obesity in the C57BL/6J mouse: physiological and molecular characteristics," Physiology and Behavior, vol. 81, no. 2, pp. 243-248, 2004.

[47] M. L. Peyot, E. Pepin, J. Lamontagne et al., “ $\beta$-cell failure in dietinduced obese mice stratified according to body weight gain: secretory dysfunction and altered islet lipid metabolism without steatosis or reduced $\beta$-cell mass," Diabetes, vol. 59, no. 9, pp. 2178-2187, 2010.

[48] J. Folch, M. Lees, and G. H. Sloane Stanley, "A simple method for the isolation and purification of total lipides from animal tissues," The Journal of Biological Chemistry, vol. 226, no. 1, pp. 497-509, 1957.

[49] C. D. Egan, "Addressing use of herbal medicine in the primary care setting," Journal of the American Academy of Nurse Practitioners, vol. 14, no. 4, pp. 166-171, 2002.

[50] C. Leduc, J. Coonishish, P. Haddad, and A. Cuerrier, "Plants used by the Cree Nation of Eeyou Istchee (Quebec, Canada) for the treatment of diabetes: a novel approach in quantitative ethnobotany," Journal of Ethnopharmacology, vol. 105, no. 1-2, pp. 55-63, 2006.

[51] M. H. Fraser, A. Cuerrier, P. S. Haddad, J. T. Arnason, P. L. Owen, and T. Johns, "Medicinal plants of cree communities (Québec, Canada): antioxidant activity of plants used to treat type 2 diabetes symptoms," Canadian Journal of Physiology and Pharmacology, vol. 85, no. 11, pp. 1200-1214, 2007.

[52] J. M. Ye, P. J. Doyle, M. A. Iglesias, D. G. Watson, G. J. Cooney, and E. W. Kraegen, "Peroxisome proliferator-activated receptor (PPAR) $-\alpha$ activation lowers muscle lipids and improves insulin sensitivity in high fat-fed rats. Comparison with PPAR- $\gamma$ activation," Diabetes, vol. 50, no. 2, pp. 411-417, 2001.

[53] J. Girard and M. Lafontan, "Impact of visceral adipose tissue on liver metabolism and insulin resistance. Part II: visceral adipose tissue production and liver metabolism," Diabetes and Metabolism, vol. 34, no. 5, pp. 439-445, 2008.

[54] M. den Boer, P. J. Voshol, F. Kuipers, L. M. Havekes, and J. A. Romijn, "Hepatic steatosis: a mediator of the metabolic syndrome. Lessons from animal models," Arteriosclerosis, Thrombosis, and Vascular Biology, vol. 24, no. 4, pp. 644-649, 2004.

[55] A. Nachar, A. Saleem, D. Vallerand et al., "Beneficial effects in the liver of antidiabetic plants used in traditional medicine by the Cree of Bay James in Canada," in Proceedings of the 10th Annual Oxford International Conference on the Science of Botanicals, Planta Medica, Mississipi, Miss, USA, 2011.

[56] H. Tilg and A. R. Moschen, "Evolution of inflammation in nonalcoholic fatty liver disease: the multiple parallel hits hypothesis," Hepatology, vol. 52, no. 5, pp. 1836-1846, 2010.

[57] J. Luther, F. Driessler, M. Megges et al., "Elevated Fra-1 expression causes severe lipodystrophy," Journal of Cell Science, vol. 124, no. 9, pp. 1465-1476, 2011.

[58] M. C. Stanton, S. C. Chen, J. V. Jackson et al., "Inflammatory signals shift from adipose to liver during high fat feeding and influence the development of steatohepatitis in mice," Journal of Inflammation, vol. 8, article 8, 2011.

[59] B. Walshe-Roussel, A. Saleem, C. Cieniak et al., "Phytochemical profiling and immunomodulatory activity of water and ethanol 
extracts from Cree of Eeyou Istchee anti-diabetic botanicals," in Joint Meeting with American Society of PharmacognosyPhytochemical Society of North America (ASP-PSNA '10), Florida, Fla, USA, 2010.

[60] Y. Zick, "Insulin resistance: a phosphorylation-based uncoupling of insulin signaling," Trends in Cell Biology, vol. 11, no. 11, pp. 437-441, 2001.

[61] J. T. Arnason, R. J. Hebda, and T. Johns, "Use of plants for food and medicine by native peoples of Eastern Canada," Canadian Journal of Botany, vol. 59, pp. 2189-2325, 1981.

[62] R. J. Marles, C. Clavelle, L. Monteleone, N. Tays, and D. Burns, Aboriginal Plant Use in Canada's Northwest Boreal Forest, UBC Press, Vancouver, Canada, 2000.

[63] A. L. Leighton, Wild Plant Use By the Woods Cree (Nihithawak) of East-Central Saskatchewan, National Museums of Canada, Ottawa, Canada, 1985.

[64] T. Efferth and E. Koch, "Complex interactions between phytochemicals. The multi-target therapeutic concept of phytotherapy," Current Drug Targets, vol. 12, no. 1, pp. 122-132, 2011. 


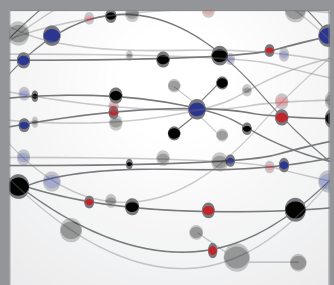

The Scientific World Journal
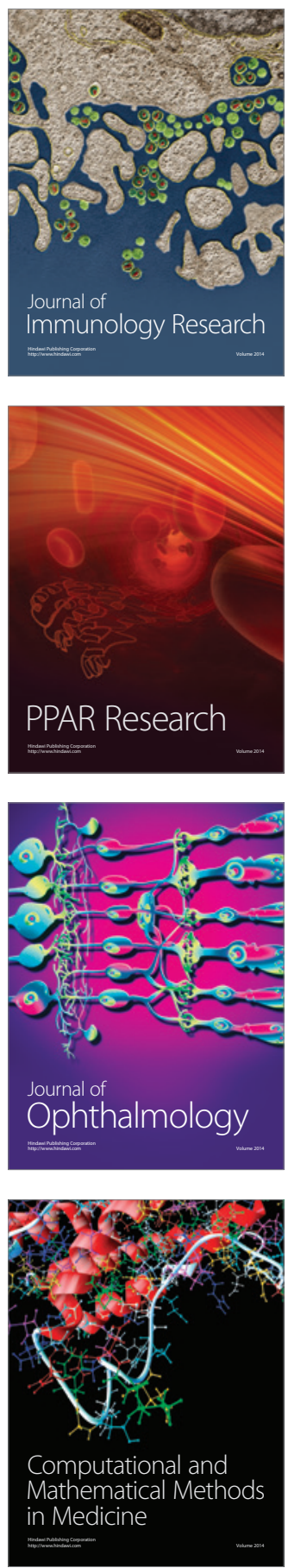

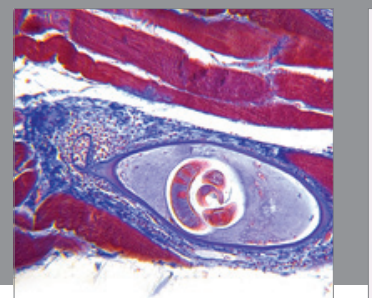

Gastroenterology

Research and Practice
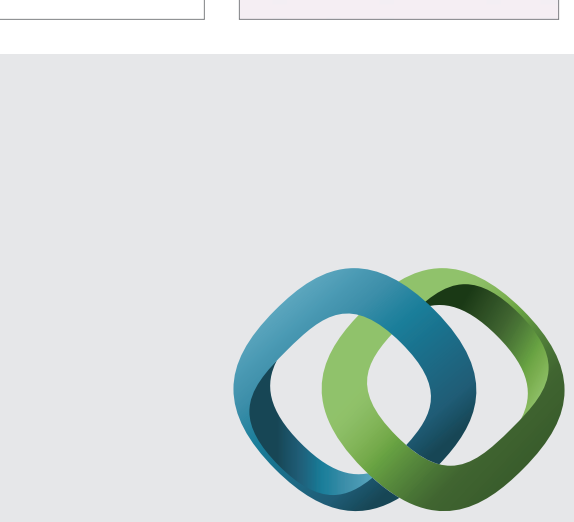

\section{Hindawi}

Submit your manuscripts at

http://www.hindawi.com
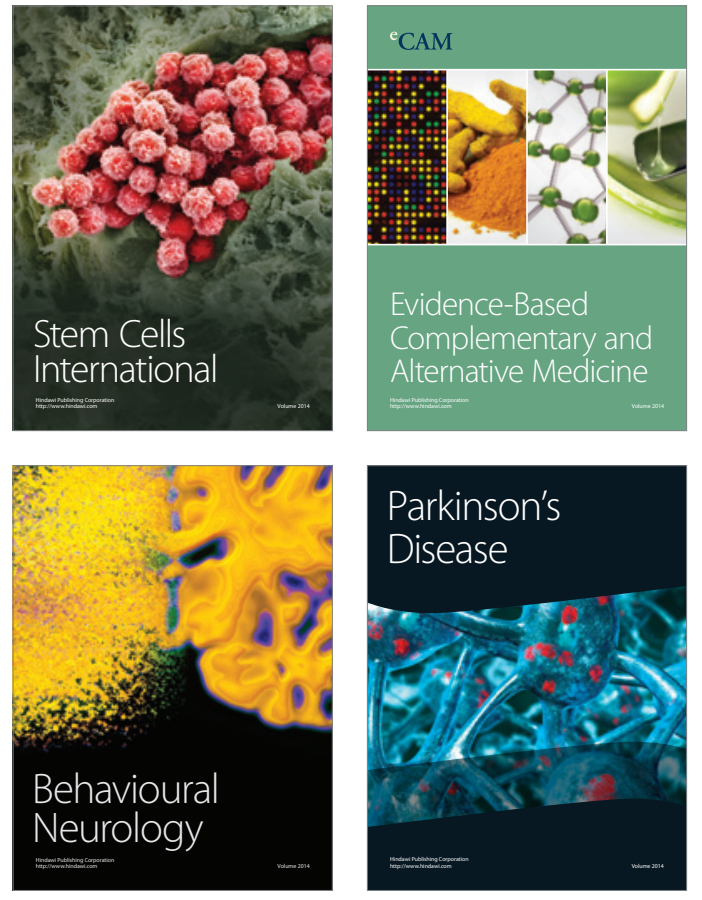
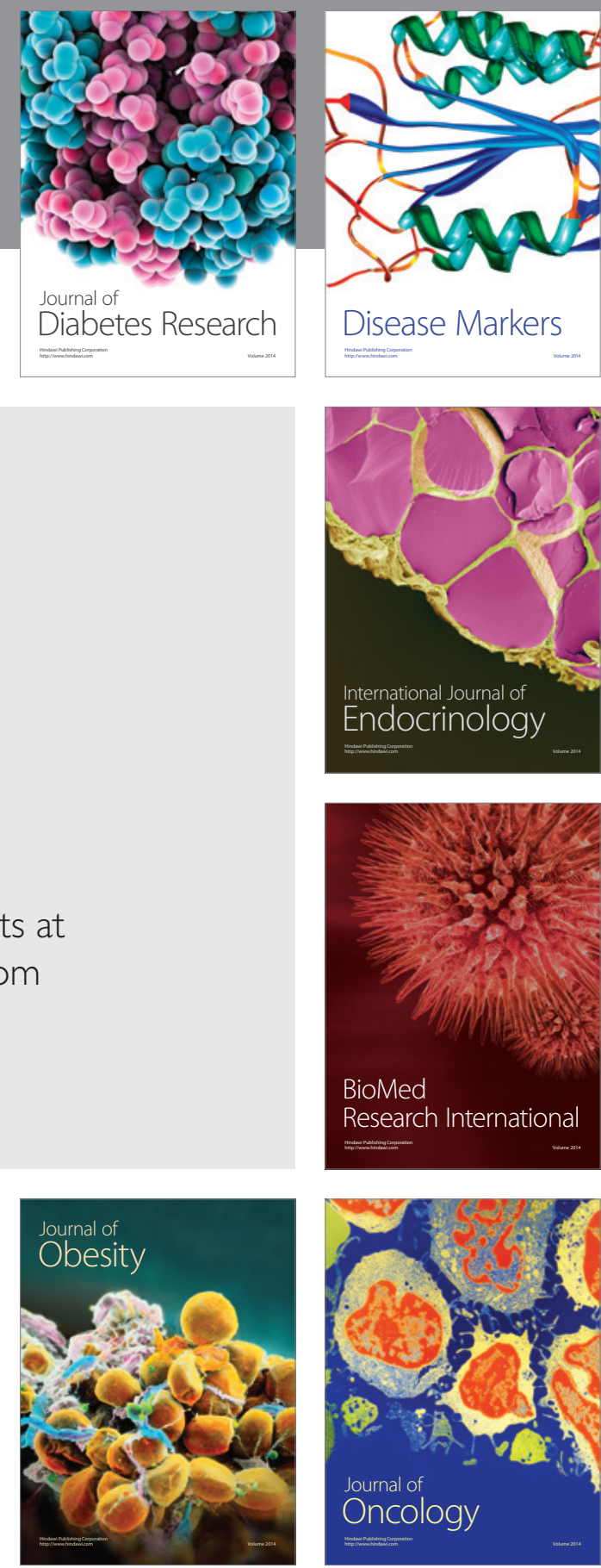

Disease Markers
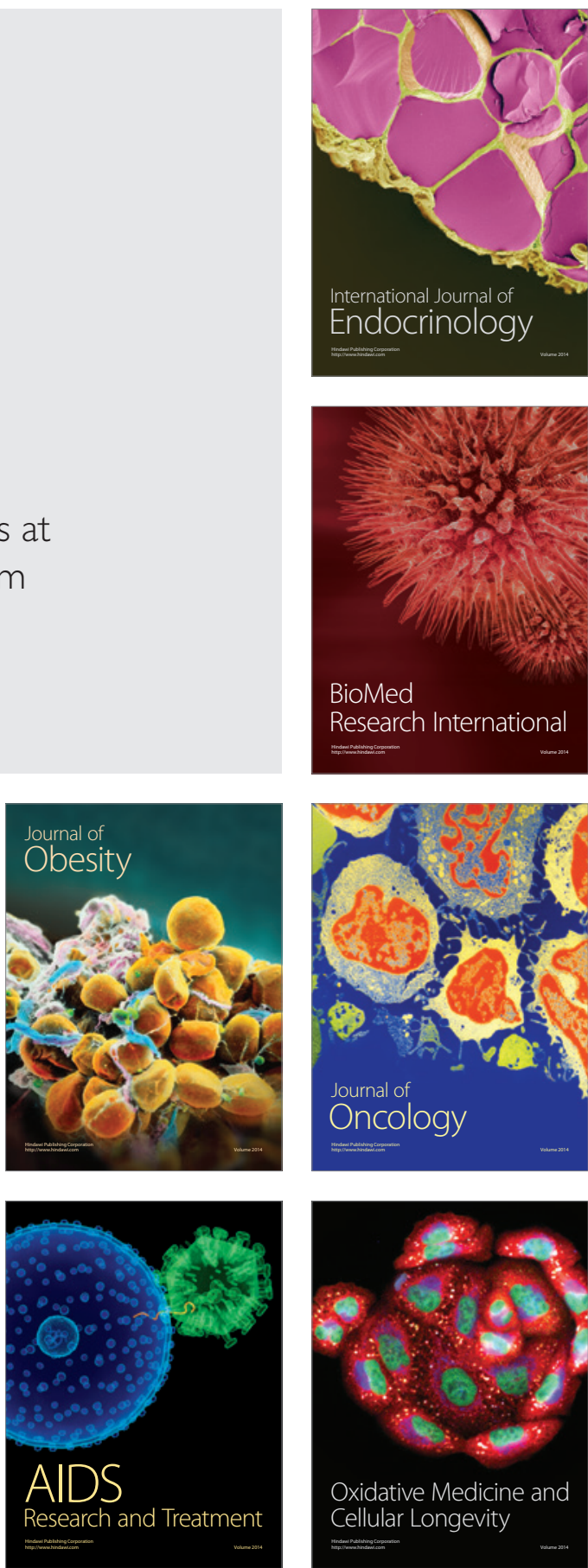